EESTI NSV TEADUSTE AKADEEMIA TOIMETISED. XIII KÖDE FUUSIKA-MATEMAATIKA- JA TEHNIKATEADUSTE SEERIA. 1964, NR. 3

ИЗВЕСТИЯ АКАДЕМИИ НАУК ЭСТОНСКОИ ССР. ТОМ ХІІ СЕРИЯ ФИЗИКО-МАТЕМАТИЧЕСКИХ И ТЕХНИЧЕСКИХ НАУК. 1964, № 3

\title{
О МОДЕЛЯХ ПРОМЕЖУТОЧНОСТИ
}

\author{
Ю. ЛУМИСТЕ,
}

\author{
кандидат физнко-математических наук
}

В работе рассматривается особый класс моделей с одним тернарным отношением - отношением промежуточности («между») -, описываемый конечной системой аксиом. Вводится понятие размерности такой модели. Двухмерные модели (плоскости промежуточности) -являются частичными плоскостями (в смысле теорин проек тивных плоскостей). Показывается, что $n$-мерные модели $n>2$ изоморфны выпуклым сбластям в линейных пространствах над линейно упорядоченными телами. Опреде ляются особые группы автоморфизмов модели промежуточности - группа движений и группа собственных движений.

1. Любое подмножество $f$ в $n$-ой декартовой степени $X^{n}=X X \ldots X X$ ( $n$ раз) множества $X$, т. е. в множестве всех упорядоченных систем $\left(x_{1}, \ldots, x_{n}\right)$ из элементов множества $X$, называется $n$-арным отнишением в $X$ (см. ['], стр. 15).

Говорят, что элементы $x_{1}, \ldots, x_{n} \in X$ находятся в отношении $f$ и пишут $f\left(x_{1}, \ldots, x_{n}\right)=1$, если $\left(x_{1}, \ldots, x_{n}\right) \in f \subset X^{n}$. Если $\left(x_{1}, \ldots, x_{n}\right) \in f=X^{n} \mid f$, то пишут $f\left(x_{1}, \ldots, x_{n}\right)=0$. Этим определяется функция с $n$ аргументами на $X$, так называемый $n$-местный предикат на $X$, которая принимает значения во множестве $E=\{0,1\}$, состоящем из двух элементов (эти элементы обозначаются условно 0 и 1 , и трактуются часто как «ложь» и «истина»). Подмножество $f \subset X^{n}$ называется определяющим . подмножеством для предиката $f\left(x_{1}, \ldots, x_{n}\right)$.

Над предикатами определены следующие 1) комбинаторные операции: фиксирование какого-нибудь одного аргумента, отождествление и перестановка каких-нибудь двух аргументов, 2) логические операции: отрицание -, конбюнкция $\Lambda$, дизоюнкция $V$ и импликация $\rightarrow$, 3) кванторы: квантор обиности $\forall$ и квантор существования $\exists$.

Следующие примеры разъясняют, в каком смысле применяются в дальнейшем эти операции и кванторы

Если заданы тернарное отношение $f \subset X^{3}$ и бинарное отношение $g \subset X^{2}$, то

1. a) $f\left(x_{1} x_{2} a\right)$, b) $f\left(x_{1} x_{2} x_{1}\right)$, c) $g\left(x_{2} x_{1}\right)$ обозначают двухместные предикаты, определяющие подмножества которых в $X^{2}$ состоят из тех пар $\left(x_{1}, x_{2}\right)$, для которых $\left.\left.\left.a\right)\left(x_{1}, x_{2}, a\right) \in f, b\right)\left(x_{1}, x_{2}, x_{1}\right) \in f, c\right)\left(x_{2}, x_{1}\right) \in g$;

2. $\overline{g\left(x_{1} x_{2}\right)}$ определяется подмножеством $\bar{g} \subset X^{2}$;

3. a) $f\left(x_{1} x_{2} x_{3}\right) \wedge g\left(x_{4} x_{5}\right)$, b) $f\left(x_{1} x_{2} x_{3}\right) \vee g\left(x_{4} x_{5}\right)$, c) $f\left(x_{1} x_{2} x_{3}\right) \rightarrow g\left(x_{4} x_{5}\right)$ эвляются 5-местными предикатами, определяюшие подмножества которых в $X^{5}$ состоят из тех систем $\left(x_{1}, \ldots, x_{5}\right)$, для которых a) $\left(x_{1}, x_{2}, x_{3}\right) \in f$ н $\left.\left(x_{4}, x_{5}\right) \in g, b\right) \quad\left(x_{1}, x_{2}, x_{3}\right) \in f$ или $\left.\left(x_{4}, x_{5}\right) \in g, c\right)$ либо $\left(x_{1}, x_{2}, x_{3}\right) \in \bar{F}$, либо $\left(x_{1}, x_{2}, x_{3}\right) \in f$ и вместе с тем $\left(x_{4}, x_{5}\right) \in g$; 
4. a) $\left.(\forall x) f\left(x_{1} x_{2} x\right), \quad b\right) .(\exists x) f\left(x_{1} x_{2} x\right)$ обозначают двухместные предикаты, определяющие подмножества которых в $X^{2}$ состоят из тех пар $\left(x_{1}, x_{2}\right)$, для которых $\left.a\right)$ при любом $x \in X$ имеет место $\left.\left(x_{1}, x_{2}, x\right) \in f, b\right)$ существует $x \in X$, такой что $\left(x_{1}, x_{2}, x\right) \in f$.

Комбинированием некоторых из этих операций можно составить, шапример, следующий предикат:

$$
\left(f\left(x_{1} x_{2} x_{3}\right) \wedge g\left(x_{1} x_{2}\right)\right) \rightarrow g\left(x_{3} x_{2}\right) .
$$

Он определяется подмножеством в $X^{3}$, состоящим из таких систем $\left(x_{1}, x_{2}, x_{3}\right)$, для которых либо 1) $\left(x_{1}, x_{2}, x_{3}\right) \in \bar{f}$ или $\left(x_{1}, x_{2}\right) \in \bar{g}$, либо 2) $\left(x_{1}, x_{2}, x_{3}\right) \in f$ и $\left(x_{1}, x_{2}\right) \in g$, и вместе с тем $\left(x_{3}, x_{2}\right) \in g$.

Если заданы $g \subset X^{n}, h \subset X^{n}$, то $g\left(x_{1} \ldots x_{n}\right) \wedge h\left(x_{1} \ldots x_{n}\right), g\left(x_{1} \ldots x_{n}\right) \bigvee$ $\forall h\left(x_{1} \ldots x_{n}\right), g\left(x_{1} \ldots x_{n}\right) \rightarrow h\left(x_{1} \ldots x_{n}\right)$ определяются, соответственно, подмножествами $g \cap h, g \cup h, \bar{g} \cup h$ в $X^{n}$.

Бинарное отношение $e \subset X^{2}$ называется единичным, если $e$ состоит из пар $(x, x) \subset X^{2}$ с совпадающими элементами. Соответствующий предикат $e\left(x_{1} x_{2}\right)$ обозначается обычно $x_{1}=x_{2}$; вместо $\overline{e\left(x_{1} x_{2}\right)}$ пишут $x_{1} \neq x_{2}$ -

2. n-арное отношение $f \subset X^{n}$ называется тождественным, если $f=X^{n}$. Соответствующий предикат $f\left(x_{1} \ldots x_{n}\right)$ на $X$, принимающий всегда значение 1 («истина»), называется тождественно истинным.

Множество, в котором задано некоторое число отношений, называется моделью. Все тождественные отношения, составленные из заданных с переходом на соответствующие предикаты и с применением вышеуказанных операций, образуют теорию модели.

3. Модель $X$ с единичным бинарным отношением и одним тернарным отношением $f \subset X^{3}$ называется неодномерной моделью промежутоности, если в ее теорию входят следующие отношения:

$1^{\circ} f\left(x_{1} x_{2} x_{3}\right) \rightarrow f\left(x_{2} x_{1} x_{3}\right)$ - симметрия по первым двум аргументам,

$2^{\circ} f\left(x_{1} x_{2} x_{3}\right) \rightarrow \overline{f\left(x_{1} x_{3} x_{2}\right)}$ - сильная антисимметрия по последним двум аргументам,

$3^{\circ} g\left(x_{1} x_{2} x_{3}\right) \bigwedge h\left(x_{1} x_{2} x_{4}\right) \rightarrow h\left(x_{1} x_{3} x_{4}\right)$ - обобщенная транзитивность; здесь

$$
\begin{aligned}
& g\left(x_{1} x_{2} x_{3}\right)=f\left(x_{1} x_{2} x_{3}\right) \vee f\left(x_{2} x_{3} x_{1}\right) \vee f\left(x_{3} x_{1} x_{2}\right), \\
& h\left(x_{1} x_{2} x_{3}\right)=g\left(x_{1} x_{2} x_{3}\right) \vee\left(x_{1}=x_{2}\right) \vee\left(x_{2}=x_{3}\right) \vee\left(x_{3}=x_{1}\right) ; \\
& 4^{\circ}\left(x_{1}-x_{3}\right) \rightarrow(\exists x) f\left(x_{1} x x_{3}\right)-\text { продолжаемость, } \\
& 5^{\circ}\left(x_{1} \neq x_{2}\right) \rightarrow(\exists x) h\left(x_{1} x_{2} x\right)-\text { неодномерность, } \\
& 6^{\circ} \overline{h\left(x_{1} x_{2} x_{3}\right)} \bigwedge f\left(x_{1} x_{4} x_{2}\right) \wedge f\left(x_{2} x_{3} x_{5}\right) \rightarrow \\
& \rightarrow\left(\exists x_{6}\right)\left(f\left(x_{1} x_{3} x_{6}\right) \wedge h\left(x_{4} x_{6} x_{5}\right)\right)-\text { аксиома Паша-Веблена (см. } \\
& \text { рис. 1). }
\end{aligned}
$$

Отношение $f$ называется тогда отношением промежуточности или отношением «между». Если $\left(x_{1}, x_{2}, x_{3}\right) \in f \subset X^{3}$, т. е. $f\left(x_{1} x_{2} x_{3}\right)=1$, то говорят, что « $x_{3}$ лежит между $x_{1}$ $u x_{2} \gg$.

Если $\left(x_{1}, x_{2}, x_{3}\right) \in h \subset X^{3}$, то $x_{1}, x_{2}$ и' $x_{3}$ назьіваются коллинеарными.

Подобные аксиомы, которые в словесной форме имеются уже у О. Веблена [2], использовал Я. Сарв [3] при построении значительной части $n$-мерной абсолютной "геометрии. Ю. Нуут [4, 5] и в

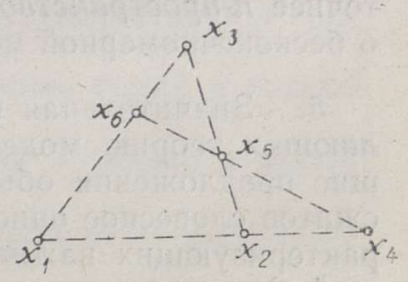

Рис. 1. 
особенности А. Хумал (Тудеберг) [6] применили уже некоторую символику. А. Хумал после тщательного анализа приводил систему аксиом к вышеуказанным 6 аксиомам.

Из более поздних работ об аксиоматизации понятия промежуточности следует отметить $[7]$, где делается ряд выводов из другой, более сложной системы аксиом, и даются некоторые указания на литературу.

4. В этом пункте строится ряд унарных отношений в $X$. При этом часто оказывается удобным подмножество $f \subset X$, определяющее одноместный предикат $f(x)$, обозначать через $\{x: f(x)\}$.

Если $a_{1}, a_{2} \in X$ являются некоторыми фиксированными точками, и $a_{1} \neq a_{2}$, то подмножество $\left\{x: h\left(a_{1} a_{2} x\right)\right\}$ в $X$ называется прямой $a_{1} a_{2}$, а подмножество $\left\{x: f\left(x a_{2} a_{1}\right)\right\}$ - полупрямой $\left.a_{1}\right) a_{2}$.

Пусть

$$
\begin{aligned}
& b\left(x_{1} x_{2} x_{3} x_{4}\right)=f\left(x_{1} \cdot x_{2} x_{4}\right) \bigvee f\left(x_{2} x_{3} x_{4}\right) \bigvee f\left(x_{3} x_{1} x_{4}\right), \\
& h^{\prime}\left(x_{1} x_{2} x_{3} x_{4}\right)=\left(\exists x, x^{\prime}\right)\left(h\left(x_{4} x x^{\prime}\right) \wedge b\left(x_{1} x_{2} x_{3} x\right) \wedge b\left(x_{1} x_{2} x_{3} x^{\prime}\right)\right) .
\end{aligned}
$$

Если $a_{1}, a_{2}, a_{3} \in X$ и $h\left(a_{1} a_{2} a_{3}\right)=0$, то множество $\left\{x: h^{\prime}\left(a_{1} a_{2} a_{3} x\right)\right\}$ называется плоскостью $a_{1} a_{2} a_{3}$, а множество $\left\{x:\left(\exists x^{\prime}\right)\left(h\left(a_{1} a_{2} x^{\prime}\right) \mid \wedge\right.\right.$ $\left.\bigwedge\left\{\left(a_{3} x x^{\prime}\right)\right)\right\}$ - полуплоскостью $\left.a_{1} a_{2}\right) a_{3}$.

Если к аксиомам $1^{\circ}-6^{\circ}$ присоединяется аксиома

$7_{(2)}^{\circ} h\left(x_{1} x_{2} x_{3}\right) \vee h^{\prime}\left(x_{1} x_{2} x_{3} x_{4}\right)$,

то соответствующая модель $X$ называется двухмерной или плоскостью промежуточности.

Пусть модель промежуточности $X$ не является двухмерной; пусть

$$
\begin{aligned}
& f^{\prime}\left(x_{1} x_{2} x_{3} x_{4}\right)=(\exists x)\left(f\left(x_{1} x_{2} x\right) \wedge f\left(x x_{3} x_{4}\right)\right), \\
& b^{\prime}\left(x_{1} x_{2} x_{3} x_{4} x_{5}\right)=f^{\prime}\left(x_{1} x_{2} x_{3} x_{5}\right) \vee f^{\prime}\left(x_{2} x_{3} x_{4} x_{5}\right) \vee f^{\prime}\left(x_{3} x_{4} x_{1} x_{5}\right) \vee f^{\prime}\left(x_{4} x_{1} x_{2} x_{5}\right), \\
& h^{\prime \prime}\left(x_{1} x_{2} x_{3} x_{4} x_{5}\right)=\left(\exists x, x^{\prime}\right)\left(h\left(x_{5} x x^{\prime}\right) \wedge b^{\prime}\left(x_{1} x_{2} x_{3} x_{4} x\right) \wedge b^{\prime}\left(x_{1} x_{2} x_{3} x_{4} x^{\prime}\right)\right) .
\end{aligned}
$$

Если $a_{1}, a_{2}, a_{3}, a_{4} \in X$ и $h\left(a_{1} a_{2} a_{3}\right) \bigvee h^{\prime}\left(a_{1} a_{2} a_{3} a_{4}\right)=0$. (некомпланарность), то множество $\left\{x: h^{\prime \prime}\left(a_{1} a_{2} a_{3} a_{4} x\right)\right\}$ называется 3-пространством $a_{1} a_{2} a_{3} a_{4}$, а множество $\left\{x:\left(\exists x^{\prime}\right)\left(h^{\prime}\left(a_{1} a_{2} a_{3} x^{\prime}\right) \bigwedge f\left(a_{4} x x^{\prime}\right)\right)\right\}$ - 3-полупространством $\left.a_{1} a_{2} a_{3}\right) a_{4}$.

Если к аксиомам $1^{\circ}-6^{\circ}$ присоединяется аксиома

$7_{(3)}^{o} h\left(x_{1} x_{2} x_{3}\right) \vee h^{\prime}\left(x_{1} x_{2} x_{3} x_{4}\right) \vee h^{\prime \prime}\left(x_{1} x_{2} x_{3} x_{4} x_{5}\right)$

то соответствующая модель $X$ называется трехмерной или 3-пространством промежуточности.

В противном случае можно продолжить указанный процесс построения новых предикатов и с их помощью ввести понятия 4-, 5- и т. д. пространств и полупространств.

Если на каком-нибудь шагу появляется аксиома $7_{(n)}^{\circ}$, заканчивающая это построение, то соответствующая модель называется конечномерной, точнее $n$-пространством променуточности. В противном случае говорят o бесконечномерной модели промежуточности.

5. Значительная часть тождественно истинных предикатов, составляющих теорию модели промежуточности, напоминает соответствующие предложения обычной элементарной геометрии. Ниже приводится сжатое словесное описание ряда нужных в дальнейшем предикатов, характеризующих важнейшие взаимные свойства точек, прямых, плоскостей, 3-пространств и т. д. в модели промежуточности. (Доказательства можно найти в ${ }^{[3]}$ или $\left[{ }^{8}\right]$; в первом они даны еще в словесном виде). 
Прямая однородна, представляет собой бесконечное (быть может, только счетное) множество точек, линейно упорядочиваемое двумя различными способами, и ее произвольная точка разделяет множество остальных точек на две полупрямые.

Плоскость однородна и представляет собой плоскость промежуточности. Если две точки прямой принадлежат плоскости, то и вся прямая принадлежит плоскости, разделяя множество остальных точек плоскости на две полуплоскости.

3-пространство однородно и представляет собой 3-пространство промежуточности. Если три точки плоскости принадлежат 3-пространству, то вся плоскость принадлежит 3-пространству, разделяя множество остальных точек 3-пространства на два 3-полупространства. Если две различные плоскости принадлежат одному 3-пространству и имеют общую точку, то они имеют общую прямую. которая содержит все их общие точки.

Аналогичными свойствами обладают 4-, 5- и т. д. пространства промежуточности (см. [3]).

В теории модели промежуточности $X$ можно определить еще следующие подмножества:

интервал: $\left(a_{1} a_{2}\right)=\left\{x: f\left(a_{1} a_{2} x\right)\right\}$, внутренность треугольника: $\left(a_{1} a_{2} a_{3}\right)=\left\{x: f^{\prime}\left(a_{1} a_{2} a_{3} x\right)\right\}$,

расиирение треугольника

а) за вершину $a_{3}: a_{1} a_{2}\left(a_{3}\right)=\left\{x: f^{\prime}\left(a_{1} a_{2} x a_{3}\right)\right\}$,

в) за сторону $\left(a_{2} a_{3}\right): a_{1}\left(a_{2} a_{3}\right)=\left\{x: f^{\prime}\left(a_{1} x a_{2} a_{3}\right)\right\}$.

Можно доказать $[3,8]$, что

$$
\left(a_{1} a_{2}\right)=\left(a_{2} a_{1}\right),\left(a_{i_{1}} a_{i_{2}} a_{i_{3}}\right)=\left(a_{1} a_{2} a_{3}\right) \text {, где }
$$

$i_{1}, i_{2}, i_{3}$ - некоторая перестановка из чисел $1,2,3$.

Прямая $a_{1} a_{2}$ является объединением точек $a_{1}$ и $a_{2}$, интервала $\left(a_{1} a_{2}\right)$, и полупрямых $\left.a_{1}\right) a_{2}$ и $\left.a_{2}\right) a_{1}$ :

$$
\left.\left.a_{1} a_{2}=\left\{a_{1}\right\} \cup\left\{a_{2}\right\} \cup\left(a_{1} a_{2}\right) \cup a_{1}\right) a_{2} \cup a_{2}\right) a_{1}
$$

Плоскость $a_{1} a_{2} a_{3}$ является объединением прямых $a_{1} a_{2}, a_{2} a_{3}$ и $a_{3} a_{1}$, внутренности треугольника $\left(a_{1} a_{2} a_{3}\right)$, всевозможных расширений этого треугольника за все вершины и стороны $\left.{ }^{8}\right]$.

Такого рода предложения имеют место и для 3-, 4- и т. д. пространств промежуточности [3].

$n$-пространство промежуточности называется непрерывным, если в нем каждая прямая, как линейно упорядоченное множество, непрерывна в смысле Дедекинда, т. е. если на ней каждое сечение является дедекин довым.

6. Теория плоскостей промежуточности имеет некоторую связь с теорией проективных плоскостей $\left[{ }^{10,11}\right]$.

Проективная плоскость -- это множество точек, определенные подмножества которого называются прямыми, и которое удовлетворяет следующим ақсиомам:

1) две различные точки принадлежат одновременно одной и только одной прямой,

2) две различные прямые пересекаются в одной и только в одной точке,

3) существуют четыре точки, любые три из которых не принадлежат одной прямой.

Если выполняются аксиомы 1) и 3), но не аксиома 2), то говорят о частичной плоскости [10]. 
Нетрудно убедиться, что плоскость промежуточности является частичной плоскостью на каждой прямой которой заданы два взаимнопротивоположных линейных порядка.

Исследование плоскостей промежуточности находится еще в начальной стадии. Также как в теории проективных плоскостей, представляет интерес выяснение взаимных связей конфигурационных теорем, возможностей ввести координаты, взятые из некоторой абстрактной алгебры, и транзитивных свойств группы автоморфизмов.

7. Множество всевозможных прямых в 3-пространстве, проходящих через фиксированную точку $a_{0}$ - так называемая (соб́ственная) 3-связка, является проективной плоскостью, если в нем под «прямыми» подразумевать подмножества прямых, принадлежащих одной плоскости.

Следующая лемма показывает, что собственная 3-связка как проективная плоскость является дезарговой плоскостью.

Л е м м а 1 (Теорема Дезарга для 3-связки). Если плоскости $a_{0} a_{1} a_{1}^{\prime}$, $a_{0} a_{2} a_{2}^{\prime}, a_{0} a_{3} a_{3}^{\prime}$, принадлежащие одному 3-пространству, попарно различны и имеют общую прямую, то прямье $a_{0} a_{1} a_{2} \cap a_{0} a_{1}^{\prime} a_{2}^{\prime}, a_{0} a_{2} a_{3} \cap a_{0} a_{2}^{\prime} a_{3}^{\prime}$. $a_{0} a_{3} a_{1} \cap a_{0} a_{3}^{\prime} a_{1}^{\prime}$ принадлежат одной плоскости. Справедливо и обратное утверждение.

Лемма доказывается в $\left(\left[{ }^{8}\right], \S 20\right)$. (Идея доказательства заимствована из [9]).

Следовательно, в 3-связке можно ввести координаты, взятые из какого-нибудь тела, используя известные построения (см. $\left.\left[{ }^{10,11}\right]\right)$.*

8. Вышеизложенное позволяет ввести координаты также в 3-пространстве промежуточности. Ниже приводятся необходимые для этого построения.

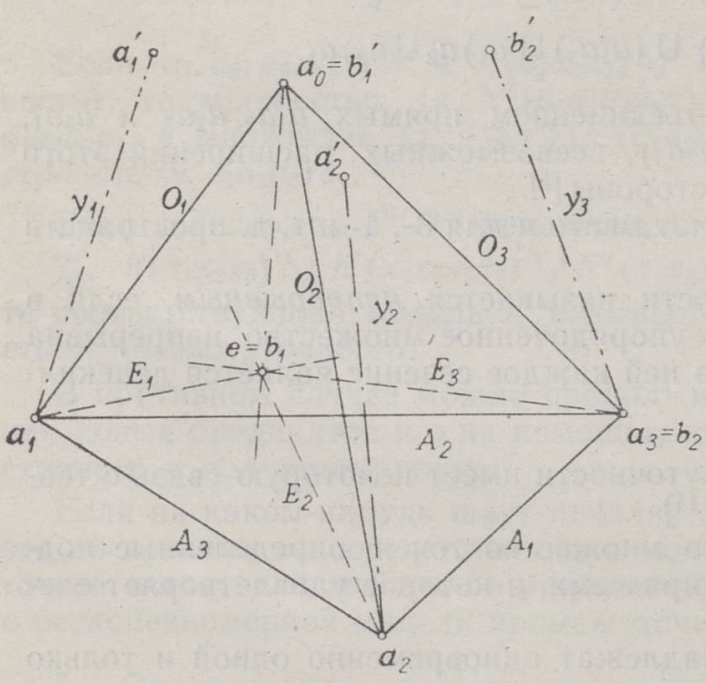

Рис. 2

Пусть $a_{0}, a_{1}, a_{2}, a_{3}-$ четыpe точки, не принадлежащие одной плоскости, и пусть $е$ является некоторой точкой во внутренности тетраэдра $\left(a_{0} a_{1} a_{2} a_{3}\right)$ (pнс. 2).

Прямые $O_{1}=a_{1} a_{0}, A_{3}=a_{1} a_{2}$, $A_{2}=a_{1} a_{3}, E_{1}=a_{1} e$ играют роль «точек» для связки $\left(a_{1}\right)$ как проективной плоскости.

По известному приему $[10,11]$ припишем «прямым» (т. е. пло скостям) пучка $\left(\dot{A}_{2}\right)$, за исключением «прямой» $A_{2} A_{3}$, некоторые символы $\xi_{2}, \eta_{2}, \ldots$ «Прямым» пучка $\left(A_{3}\right)$, за исключением «прямой» $A_{3} A_{2}$, припишем символы $\xi_{3}, \eta_{3} \ldots$, причем так, чтобы «прямые» пучков $\left(A_{2}\right)$ и $\left(A_{3}\right)$, пересекающиеся в некоторой «точке» $y_{1}$ «прямой»

- Представляет ннтерес выяснить, не является ли описанная собственная 3-связка в. 3-пространстве промежуточности проективной упорядоченной плоскостью в смысле P. Бэра $\left[{ }^{12}\right]$. 
$O_{1} E_{1}$, были обозначены одинаковыми символами (т. е. чтобы для них $\left.\xi_{2}=\xi_{3}=\xi, \eta_{2}=\eta_{3}=\eta, \ldots\right)$. «Прямым» $A_{2} O_{1}, A_{3} O_{1}$ припишем символ 0 , «прямым» $A_{2} E_{1}, A_{3} E_{1}$ - символ 1 , «прямой» $A_{2} A_{3}-$ символ $\infty$

Через произвольную «точку» $X_{1}=a_{1} x$ проходит «прямая» $A_{2} X_{1}$ с сим волом छे и ирямая» $A_{3} X_{1}$ с символом $\xi_{3}$. «Точке» $X_{1}$ припишем координаты $\left(\xi_{2}, \xi_{3}\right)$ и обозначим $X_{1}=\left(\xi_{2}, \xi_{2}\right)$. Очевидно, $O_{1}=(0,0), E_{1}=(1,1)$. $y_{1}=(\xi, \xi)$.

Точно так же можно ввести координаты в связке $\left(a_{2}\right)$ на базе «точек» $\mathrm{O}_{2}=a_{2} a_{0}, A_{3}=a_{2} a_{1}, A_{1}=a_{2} a_{3}, E_{2}=a_{2} e$, причем так, что «прямым» пучка $\left(A_{3}\right)$ приписываются те же самые символы $\xi_{3}, \eta_{3}, \ldots$, которые они получили в связке $\left(a_{1}\right)$. Произвольная «точка» $X_{2}=a_{2} x$ получает коор динаты $\left(\xi_{2}, \xi_{1}\right)$.

Для связки $\left(a_{3}\right)$ остается только проверить, что «прямые» пучков $\left(A_{1}\right)$ и $\left(A_{2}\right)$ с одинаковыми символами пересекаются в «точке» $y_{3}$ «прямой» $\mathrm{O}_{3} E_{3}$ (где $\mathrm{O}_{3}=a_{3} a_{0}, E_{3}=a_{3} e$ ).

Для этого нужно применить следующую лемму, доказательство которой дано в $\left(\left[{ }^{8}\right], \S 20\right)$.

Л ем м а 2. Если среди точек $a_{1}, a_{1}^{\prime}, a_{2}, a_{2}, b_{1}, b_{1}^{\prime}, b_{2}, b_{2}^{\prime}$ любые три не принадлежат одной прямой $u$ 1) $\left.a_{2}^{\prime} \in a_{1} a_{1}^{\prime} a_{2}, 2\right) b_{1}, b_{2} \notin a_{1} a_{1}^{\prime} a_{2}$, 3) $b_{1}^{\prime} \in a_{1} a_{1}^{\prime} b_{1} \cap a_{2} a_{2}^{\prime} b_{1}$, 4) $b_{2}^{\prime} \in a_{1} a_{1}^{\prime} b_{2} \cap a_{2} a_{2}^{\prime} b_{2}$, 5) $a_{1} a_{1}^{\prime} a_{2} \cap b_{1} b_{1}^{\prime} b_{2} \neq \varnothing$, то $b_{1}, b_{2}, b_{1}^{\prime}, b_{2}^{\prime}$ принадлежат одной плоскости.

Применение этой леммы состоит в следующем (рис. 2). Пусть некоторые «прямые» пучков $\left(A_{2}\right)$ и $\left(A_{3}\right)$ пересекаются в «точке» $y_{1}=a_{1} a_{1}^{\prime}$. принадлежащей «прямой» $O_{1} E_{1}$. Пусть в пучке $\left(A_{1}\right)$ выбрана «прямая», пересекающая «прямую» $\mathrm{O}_{2} E_{2}$ в «точке» $y_{2}=a_{2} a_{2}$. принадлежащей «прямой» $y_{1} A_{3}$. Тогда «прямые» $A_{2} y_{1}$ и $A_{3} y_{1}$, а также $A_{3} y_{2}$ и $A_{1} y_{2}$ имеют одинаковые символы. Но ввиду того, что плоскости $A_{2} V_{1}$ и $A_{3} y_{2}$ совпадают, одинаковые символы получают также «прямые» $A_{2} y_{1}$ и $A_{1} y_{2}$.

Из леммы 2 следует теперь, что эти «прямые» $A_{2} V_{1}$ и $A_{1} y_{2}$, действительно пересекаются в «точке» $y_{3}=a_{3} b_{2}^{\prime}$, принадлежащей «прямой» $\mathrm{O}_{3} E_{3}$. (Нужно взять $b_{1}=e, b_{1}^{\prime}=a_{0}, b_{2}=a_{3}$ ).

Координаты точки $x \in X$ вводятся теперь следующим образом. Из вышеизложенного следует, что пары координат $\left(\xi_{2}, \xi_{3}\right),\left(\xi_{3}, \xi_{1}\right),\left(\xi_{1}, \xi_{2}\right)$ прямых $a_{1} x, a_{2} x, a_{3} x$ в соответствующих связках $\left(a_{1}\right),\left(a_{2}\right),\left(a_{3}\right)$ состав лены только из трех символов $\xi_{1}, \xi_{2}, \xi_{3}$. Тройка этих символов и приписывается точке $x$, обозначая $x=\left(\xi_{1}, \xi_{2}, \xi_{3}\right)$

Точке $x_{\infty}$, принадлежащей плоскости $a_{1} a_{2} a_{3}$ и поэтому выпадающей из указанной конструкции, приписывается тройка символов $\left(\xi_{1} / 0, \xi_{2} / 0\right.$, $\left.\xi_{3} / 0\right)$, где $\left(\xi_{1}, \xi_{2}, \xi_{3}\right)$ - координаты какой-нибудь точки на прямой $a_{0} x$

9. Аналогичным путем можно ввести координаты также в $n$-пространство промежуточности.

Те орем а 1. Произвольное п-пространство промежуточности при $n>2$ изоморфно выпуклой области в $n$-мерном линейном пространстве $L_{n}$ над линейно упорядоченным телом $K$, в котором среди $x_{1}, x_{2}, x_{3} \in I_{n}$ элемент $x_{3}$ сиитается лежащим между $x_{1}$ и $x_{2}$ в точности тогда, если суиествует $\lambda \in K, 0<\lambda<1$, так что

$$
x_{3}=\lambda x_{1}+(1-\lambda) x_{2}
$$


Классификация $n$-пространств промежуточности при $n>2$ сводится. следовательно, к классификации линейно упорядоченных тел (теория которых, кстати, находится еще в начальной стадии (см., например $\left[{ }^{13}\right]$ ).

Ниже дается эскиз доказательства теоремы. Для простоты ограничиваемся случаем $n=3$.

Известно, что в случае дезарговой проективной плоскости можно в множестве координатных символов ввести операции сложения и умножения, так что это множество становится телом $K\left[1{ }^{10},{ }^{11}\right]$.

В случае 3-пространства промежуточности можно это сделать в каждой собственной 3-связке $\left(a_{1}\right),\left(a_{2}\right),\left(a_{3}\right)$, причем оказывается, что сложение и умножение символов не зависят от выбора связки, с помощью которой они определены.

Каждую плоскость можно представить одним линейным уравнением $\alpha_{1} \xi_{1}+\alpha_{2} \xi_{2}+\alpha_{3} \xi_{3}+\alpha_{0}=0$ с коэффициентами в теле $K$, которому удовлетворяют координаты всех точек, принадлежащих плоскости.

Каждой точке $x$ соответствует упорядоченная тройка $\left(\xi_{1}, \xi_{2}, \xi_{3}\right)$ элементов из $K$, но обратно, не каждая тройка элементов из $K$ определяет точку в 3-пространстве промежуточности (прямые $\left(\xi_{2}, \xi_{3}\right),\left(\xi_{3}, \xi_{1}\right)$, $\left(\xi_{1}, \xi_{2}\right)$ в связках $\left(a_{1}\right),\left(a_{2}\right),\left(a_{3}\right)$ могут не пересекаться). Если тройка $\left(\xi_{1}, \xi_{2}, \xi_{3}\right)$ не соответствует какой-нибудь точке $x$, то ей приписывается некоторый новый объект, называемый несобственной тоикой. (Несобственную точку можно при желании представить в виде связки (в общем случае несобственной) - множества прямых, попарно принадлежащих одной плоскости).

С помощью линейных уравнений определяются: принадлежность несобственной точки данной плоскости или прямой, несобственные плоскости и прямые. Совокупность всех точек и всех несобственных точек представляет собой 3 -мерное проективное пространство $P_{\mathrm{s}}(K)$ над $K\left[{ }^{14}\right]$, в которое можно ввести однородные координаты $\left(\varrho_{0}, \varrho_{i}\right)$, так что $\varrho_{0} \xi_{i}=Q_{i}, i=1,2,3$.

Множество собственных точек (точек 3-пространства промежуточности) должно быть выпуклым относительно отношения промежуточности, иначе не были бы удовлетворены аксиомы $1^{\circ}-6^{\circ}$. Поэтому множество несоб́ственных точек содержит по крайней мере одну несобственную плоскость.

В $P_{3}(K)$ можно сделать такое проективное преобразование

$$
\varrho_{\lambda}^{\prime}=\sum a_{\lambda \mu} Q_{\mu}, \quad \lambda, \mu=0,1,2,3
$$

при котором эта несобственная плоскость становится плоскостью $\mathrm{e}_{0}^{\prime}=0$.

Каждой точке 3-пространства промежуточности соответствует сис-

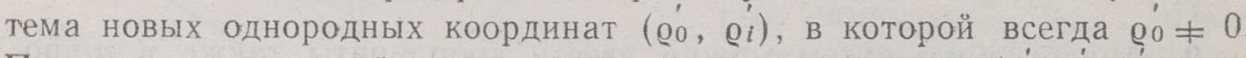
Поэтому можно перейти к неоднородным координатам $\left(\xi_{1}^{\prime}, \xi_{2}^{\prime}, \xi_{3}^{\prime}\right)$, так что $\varrho \dot{\xi}_{i}^{\prime}=\varrho \dot{i}$. Здесь $\xi_{i}^{\prime}$ являются вполне определенными элементами из тела $K$.

Множество упорядоченных троек $\bar{x}=\left(\xi_{1}^{\prime}, \xi_{2}^{\prime}, \xi_{3}^{\prime}\right)$ элементов из тела $K$ можно известным образом превратить в 3-мерное линейное пространство над телом $K$. Прямая, определяемая точками $\bar{o}=(0,0,0)$ и $\overrightarrow{e_{1}}=(1,0,0)$, состоит из точек $\bar{l}=\overrightarrow{0}+\lambda \overrightarrow{e_{1}}, \lambda \in K$. Так как прямая $\overrightarrow{o e_{1}}$ с выбранным на ней направлением от $\bar{o}$ к $\bar{e}_{1}$ является линейно упорядоченным множеством в 3-пространстве промежуточности, то и в тело $K$ 
вводится линейный порядок элементов. При этом точка $\bar{x}_{3}$ лежит между $\bar{x}_{1}$. н $\vec{x}_{2}$ в точности тогда, если для соответствующей тройки найдется $\lambda \in K, 0<\lambda<1$, так что

$$
\bar{x}_{3}=\lambda \bar{x}_{1}+(1-\lambda) \bar{x}_{2}
$$

\section{Әтим доказательство заканчивается.}

10. При классификации проективных плоскостей, как известно, важную роль играют свойства группы автоморфизмов плоскости (груп пы коллинеации) [11].

Аналогичные проблемы можно ставить в случае плоскостей и пространств промежуточности. Особый интерес представляет выделение гаких подгрупп автоморфизмов, которые могут быть названы группами движений.

Подмножество в $n$-пространстве промежуточности, являющееся объединением точки $a_{1}$, полупрямой $\left.a_{1}\right) a_{2}$, полуплоскости $\left.a_{1} a_{2}\right) a_{3}$, , $\kappa$-полупространства $\left.a_{1} \ldots a_{k}\right) a_{k+1}$, называется $\kappa$-репером и обозначается $R\left(a_{1} a_{2} \ldots a_{k+1}\right)$.

Группа автоморфизмов $n$-пространства промежуточности называется группой движений $\Omega$ (әруппой собственньх движений $\Omega_{0}$ ), если она действует просто-транзитивно на множестве всех $n$-реперов $((n-1)$ реперов).

Можно говорить о теории пары $(X, \Omega)$, подразумевая под этим совокупность тождественно истинных предикатов на декартовых произведениях, составленных из $X$ и $\Omega$, которые можно формулировать в терминах предиката промежуточности $f\left(x_{1} x_{2} x_{3}\right)$, предиката представления $x \circ \varphi=y$ и групповой операции $\varphi \psi=\chi ; x_{1}, x_{2}, x_{3}, x, y \in X, \varphi, \psi, \chi \in \Omega$

Tе орема 2. Eсли n-пространство промежуточности $X$ является непрерывным, то теория пары $(X, \Omega)$ совпадает с п-мерной абсолютной геометрией.

Доказательство теоремы при $n=2$ и $n=3$ содержится в [8], где элементарная абсолютная геометрия строится на основании 8 аксиом на отношение «между» (аксиомы $1^{\circ}-6^{\circ}$, аксиома $7_{(2)}^{\circ}$ или $7_{(3)}^{\circ}$, аксиома непрерывности (Дедекинда)), 7 аксиом на предикат представления и 4 групповых аксиом.

Теория пары $(X, \Omega)$ без требования непрерывности еще слабо развита. Здесь основные трудности связаны со следующей проблемой, которая формулируется ниже для случая $n=2$.

В группе движений $\Omega$ существует элемент $\varphi$, действие которого на $X$ переводит репер $R\left(a_{1} a_{2} a_{3}\right)$ в репер $R^{\prime}\left(a_{2} a_{1} a_{3}\right)$. Переходит ли тогда $a_{2}$ в $a_{1}$ ?

Если выполняется аксиома непрерывности, то положительный ответ на этот вопрос был дан Б. Н. Делоне $\left[{ }^{15}\right]$. В общем случае вопрос, насколько нам известно, еще открыт.

В заключение отметим интересный результат Д. Гильберта $\left[{ }^{16}\right]$, относящнйся к теории пары $\left(X, \Omega_{0}\right)$ при $n=2$. Оказывается, что в теории этой пары из равнобедренности треугольника не следует конгруэнтность углов при основании. Соответствующую модель Гильберта, построенную для аксиоматики несколько другого характера, наиболее естественно трактовать именно в терминах теории пары $\left(X, \Omega_{0}\right)$. 
1. Қ урош А. Г., Лекции по общей алгебре, М., 1962.

2. Veble n O., Trans. Amer. Math. Soc., 5, 343-384 (1904).

3. S a rv J., Geomeetria alused, Acta et Comment. Univ. Tartuensis (Dorpatensis), A 19, nr. 4 (1931).

4. Nu u.t J., Topologische Grundlagen des Zahlbegriffs, Acta et Comment. Univ. Tartuensis (Dorpatensis), A 15, Nr. 5 (1929).

5. Nuut J., Einige Bemerkungen über Vierpunktaxiome, Acta et Comment. Univ. Tartuensis (Dorpatensis), A 23, Nr. 4 (1932).

6. Tudeberg A., Niber die Beweisbarkeit einiger Anordnungsaussagen in geometrischen Axiomensystemen, Acta et Comment. Univ. Tartuensis (Dorpatensis), A 26, Nr. 6 (1934).

7. Ha sh i moto J., Osaka Math. Journ., 10, 147-158 (1958).

8. Lumiste U., Geomeetria alused I, Tartu (TRU rotaprint), 1963.

9. Schur F., Grundlagen der Geometrie, Leipzig, 1909.

10. Скорн яков Л. А., Успехи матем. наук, вып. 6(46), 6, $112-154$ (1951).

11. Холл М.. Теория групп и проективные плоскости. В кн.: Теория групп, М., 1962.

12. Б э Р Р., Проективное унорядочение пространства (гл. III, добавл. III). В кн.: Линейная алгебра и проективная геометрия, М., 1955.

13. S z ele T., Proc. Amer. Math. Soc., 3, 410-413 (1952).

14. Х.одж В., Пи до Д., Проективное пространство (ч. II). В кн.: Методы алгебраической геометрии, 1, М., 1954.

15. Делоне Б. Н., Краткое изложение доказательства непротиворечивости планиметрии Лобачевского, М., 1953.

16. Ги ль бе р т Л., Основания геометрии, М.-Л., 1948 (добавление II).

Тартуский

государственный университет
Поступила в редакцию

3. XII 1963

\section{VAHELSEISU MUDELITEST}

\section{U. Lumiste}

\section{Resümee}

Töös vaadeldakse eritüüpi mudeleid $X$ ühe ternaarse suhtega $f\left(x_{1} x_{2} x_{3}\right)$, mida nimetatakse vahelseisu suhteks. $f\left(x_{1} x_{2} x_{3}\right)$ tõesuse puhul köneldakse, et $x_{3}$ on $x_{1}$ ja $x_{2}$ vahel. Vahelseisu mudelite klassi kirjeldavad aksioomid $1^{\circ}-6^{\circ}$, mis on pärit A. Humala (Tudebergi) artiklist [6] (hilisemate sellesuunaliste tööde kohta vt. [7]). Täiendava aksioomi $7_{(n)}^{\circ}$ abil tuuakse sisse vahelseisu mudeli $X$ mõõtme mõiste.

Kahemõõtmelised mudelid $X$ (vahelseisu tasandid) osutuvad osalisteks tasanditeks (projektiivsete tasandite teooria seisukohalt; vt. $\left[{ }^{10}\right]$ ).

Vahelseisu mudelis $X$ mõõtmega $n>2$ osutub iga 3 -sidum (üht punkti läbivate sirgete hulk 3-mõõtmelises alammudelis) kui projektiivne tasarid Desargues'i tasandiks. Selle alusel formuleeritakse (ja tōestatakse $n=3$ puhul) järgmine teoreem:

Iga vahelseisu mudel $X$ mõõtmega $n>2$ on isomorfne kumera piirkonnaga $n$-mõõtmelises lineaarruumis üle järjestatud kaldkorpuse.

Tuuakse sisse $k$-reeperi mõiste, mille abil defineeritakse peegeldusliikumiste (pärisliikumiste) rühm $\Omega\left(\Omega_{0}\right)$ kui $n$-mõõtmelise vahelseisu mudeli $X$ automorfismide rühm, mis toimib lihttransitiivselt $n$-reeperite $((n-1)$-reeperite) hulgal. Kui sirged mudelis $X$ järjestatud hulkadena on pidevad Dedekindi mõttes, siis paari $(X, \Omega)$ teooria ühtib $n$-mõõtmelise absoluutse geomeetriaga. Seatakse mõningad probleemid paari $(X, \Omega)$ teooria kohta mittepideval juhul. 


\section{ON MODELS OF BETWEENNESS}

\section{U. Lumiste}

\section{Summary}

In this paper a special kind of models with one ternary relation $f\left(x_{1} x_{2} x_{3}\right)$, which is called a relation of betweenness is considered: when $f\left(x_{1} x_{2} x_{3}\right)$ is true; we say that $x_{3}$ is between $x_{1}$ and $x_{2}$. The class of models of betweenness $X$ is described by a system of axioms $1^{\circ}-6^{\circ}$ taken from A. Humal's (Tudeberg's) article ${ }^{[6]}$ (more recent works of this kind $\left.\left.s .{ }^{7}\right]\right)$. The notion of dimension for model $X$ of betweenness is introduced with a complementary axiom $7_{(n)}^{\circ}$

Two-dimensional models $X$ (planes of betweenness) prove to be partial planes (in the sense of the theory of projective planes; s. $\left.\left[{ }^{10}\right]\right)$.

In the model of betweenness with dimension $n>2$ each 3 -bundle (a set of straight lines in a 3-dimensional submodel that passes through a fixed point) as a projective plane turns out to be a desarguesian plane. On these grounds a formulation (and proof in the case $n=3$ ) for the following theorem is given:

Every model of betweenness $X$ with dimension $n>2$ is isomorphic with a convex region in the n-dimensional linear space over an ordered skew field.

The notion of $k$-frame is introduced, by which a group of reflections-movements $\Omega$ (group of proper movements $\Omega_{0}$ ) is defined as a group of automorphisms of model $X$. acting simple-transitively on the set of all $n$-frames $((n-1)$-frames). When the straight lines in the model $X$ as ordered sets are continuous in the sense of Dedekind, the theory of couple $(X, \Omega)$ coincides with $n$-dimensional absolute geometry. Some problems are raised about the theory of couple $(X, \Omega)$ (couple $\left(X, \Omega_{0}\right)$ ) in the non-continual case.

Tartu State University

Received

Dec. 3rd, 1963 